\title{
Canadian Meteor Orbit Radar (CMOR)
}

\author{
A. R. Webster ${ }^{1,2}$, P. G. Brown ${ }^{1}$, J. Jones ${ }^{1}$, K. J. Ellis ${ }^{3}$, and M. Campbell-Brown ${ }^{4}$ \\ ${ }^{1}$ Department of Physics, The University of Western Ontario, Canada \\ ${ }^{2}$ Department of Electrical and Computer Engineering, The University of Western Ontario, Canada \\ ${ }^{3}$ Defence Science and Technology Organisation, Edinburgh, SA 5111, Australia \\ ${ }^{4}$ European Space Agency, ESTEC, SCI-SB, Keplerlaan 1, NL-2201 AZ Noordwijk ZH, The Netherlands
}

Received: 30 September 2003 - Published in Atmos. Chem. Phys. Discuss.: 18 February 2004

Revised: 16 April 2004 - Accepted: 28 April 2004 - Published: 7 May 2004

\begin{abstract}
The radar system described here (CMOR) comprises a basic 5-element receiving system, co-located with a pulsed transmitter, specifically designed to observe meteor echoes and to determine their position in space with an angular resolution of $\sim 1^{\circ}$ and a radial resolution of $\sim 3 \mathrm{~km}$. Two secondary receiving sites, a few $\mathrm{km}$ distant and arranged to form approximately a right angle with the base station, allow the determination of the velocity (speed and direction) of the meteor that, together with the time of occurrence, lead to an estimate of the orbit of the original meteoroid. Some equipment details are presented along with a method used to determine the orbits. Representative echoes are shown and observations on the 2002 Leonid shower presented.
\end{abstract}

\section{Introduction}

The idea behind CMOR is to measure the characteristics of meteor echoes in such a way that the orbital parameters of the incident meteoroid can be determined for many echoes seen at the main radar site. The use of two outlying receiving antennas, and the relaying of the signals from these to the main site allows a good estimate of the parameters from the difference in time of observation at the 3 sites. Because of the geometry involved, only a fraction (perhaps $25 \%$ ) of the echoes at the main site will be accompanied by echoes from both of the other two. Nevertheless, since several thousand echoes per day are observed typically at the main site, many estimates of meteor orbits are available.

The main site in fact has 3 separate radars, operating at 3 different frequencies but otherwise identical, as part of a campaign to shed light on the initial radius problem (Campbell-Brown and Jones, 2003). Operation of all the radars is automatic and monitored remotely from the main

Correspondence to: A. R. Webster

(awebster@eng.uwo.ca) laboratory at the University of Western Ontario. The two outlying are coupled with one of the radars at an operating frequency of $29.85 \mathrm{MHz}$; the details are shown in Table 1.

\section{The equipment}

\subsection{The main system}

The transmitter is located at the main site and the receiving system consists of 5 spaced antennas arranged as two threeelement arrays along orthogonal axes with the center antenna common to both (Fig. 1). The key point of this arrangement is that the separation between the center antenna and the two outer antennas in each array differ by one half-wavelength $(\lambda / 2)$. This allows an accurate unambiguous estimate of the angle-of-arrival ( $\xi$ ) relative to the array axis (Fig. 2) from the two estimates in Eq. (1), the first giving an accurate but multivalued estimate and the second an unambiguous, less accurate, value which allows selection of the correct estimate.

$\sin \xi=-\frac{\lambda}{2 \pi} \frac{\left(\phi_{10}-\phi_{20}\right)}{\left(d_{1}+d_{2}\right)}=-\frac{\lambda}{2 \pi} \frac{\left(\phi_{10}+\phi_{20}\right)}{\left(d_{1}-d_{2}\right)}$

Conversion to elevation and azimuthal angles is straightforward and the array dimensions used lead to an accuracy of $\pm 1.0^{\circ}$ for elevation angles above $30^{\circ}$ and signal:noise greater than $10 \mathrm{~dB}$; the principle is discussed in more detail by Jones et al. (1998). The antennas are arranged at a height above ground that gives all-round coverage. Echoes from the main system for 19 November 2002 are shown in Fig. 3 in which the "dead-time" from the pulse repetition frequency used is clearly visible and the distribution is entirely consistent with the antenna radiation pattern and the general radiant distribution. 
Table 1. The radar details.

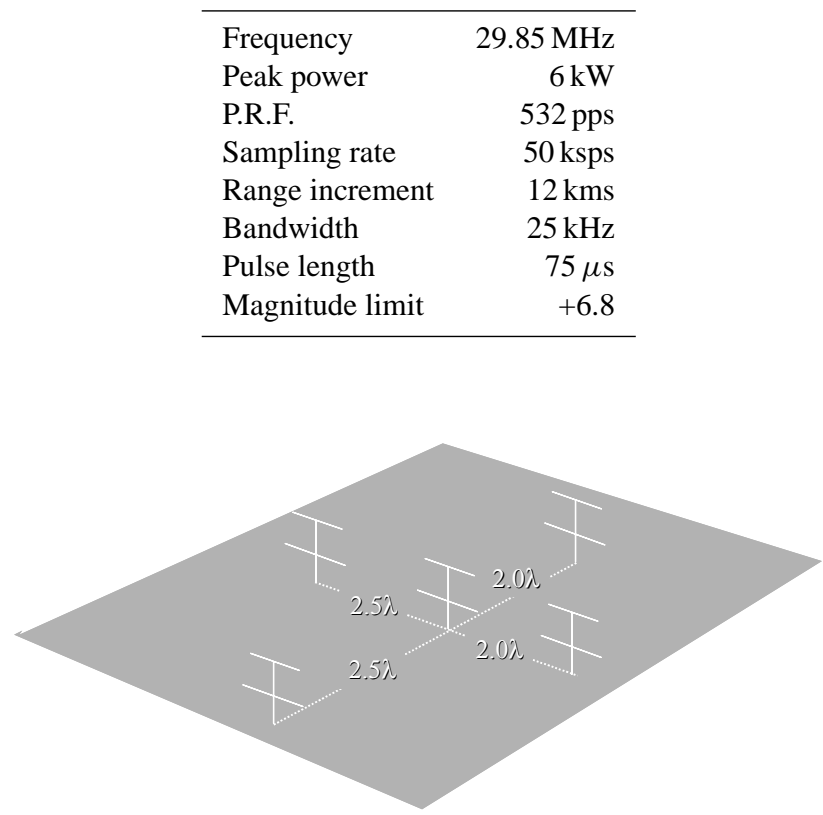

Fig. 1. The layout of the main site receiving antenna system consisting of two orthogonal three-element arrays of vertically pointing Yagi antennas.

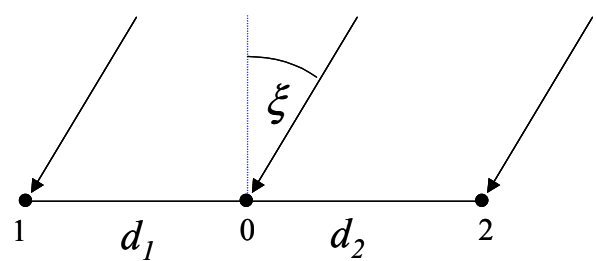

Fig. 2. The 3-element array used to determine the angle-of-arrival, $\xi$; the separations used are $d_{1}=2.0 \lambda$ and $d_{2}=2.5 \lambda$.

\subsection{The outlying receivers}

In order to determine the orbit of individual meteoroids, additional information is needed over and above the accurate position in space of the resultant meteor. One approach is to establish two extra remote receiving sites and determine the time of observation of some characteristic part of the meteor echo at each of the three sites as proposed by T. R. Kaiser (Hawkins, 1964). As a compromise between high rate of simultaneous observation and high accuracy in the final answer, which are to some extent in conflict, a right-angle arrangement with separation of $\sim 8 \mathrm{~km}$ is generally taken as optimum. Practical considerations regarding site location do come into play and the values used here are shown in Fig. 4, and are close to the optimum.

Antennas identical to those at the receiving site were used at the remote sites. The output at $29.85 \mathrm{MHz}$ was translated

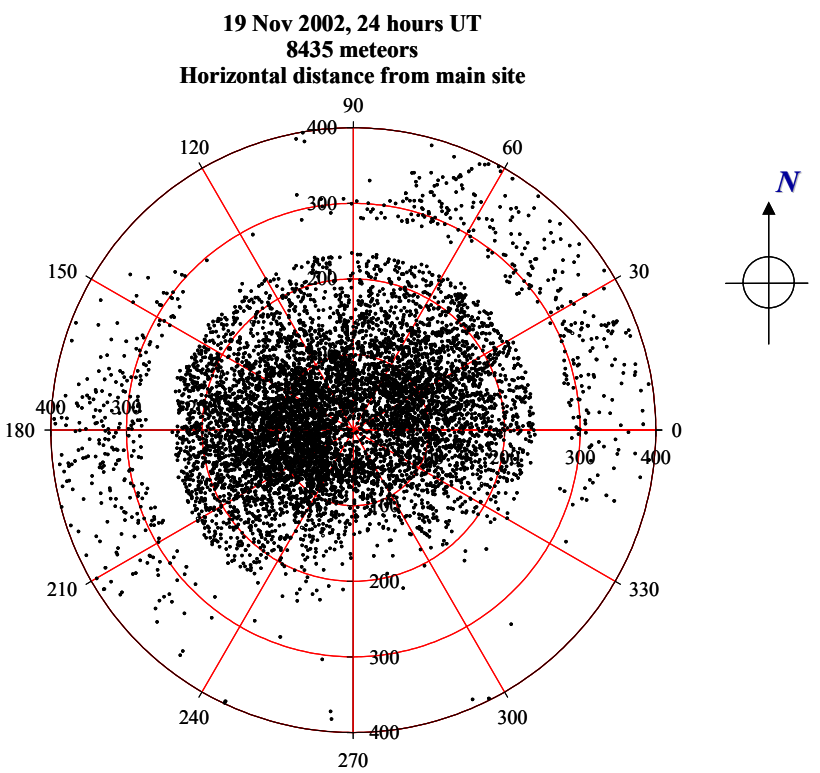

Fig. 3. The distribution of echoes on the $100 \mathrm{~km}$ surface. The distribution is consistent with the antenna radiation patterns and the overall radiant distribution. Note the sharp boundary of the blanking period associated with the "next transmitted pulse" which is consistent with the accuracy $\left( \pm 1.0^{\circ}\right)$ in angle-of-arrival (AOA) quoted.

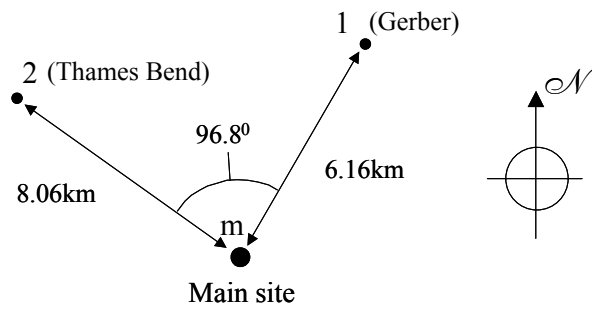

Fig. 4. The geographical layout of the 3-station system showing the main site, $\mathrm{m}$, and the two outlying sites, 1 and 2 .

up to $435 \mathrm{MHz}$ and transmitted to the main site where it was heterodyned back down to the lower frequency and inserted into the main receiver; the receiver was designed with 7 separate channels. Software routines in the main system compare the echoes and their timing to allow determination of the orbit in cases where echoes are received at all three locations.

\section{Derivation of orbital parameters}

In order to arrive at an estimate of the orbital parameters of the meteoroid in space before it interacts with the Earth, the orientation and speed of the observed meteor need to be determined. Additionally, corrections for the influence of the Earth, its orbital and rotational speeds and the effects of its gravity and atmosphere (in decelerating the meteoroid) need to be made in the final estimate; the first three 


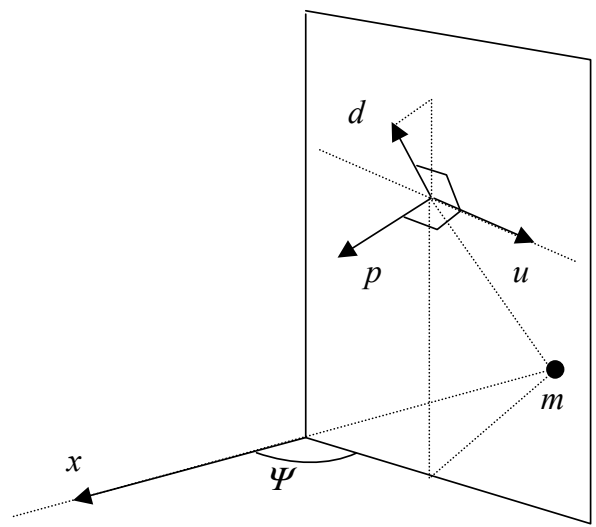

Fig. 5. Determination of the meteor direction vector $\boldsymbol{u}$ form the unit vector $\boldsymbol{d}$ from the main station and the unit vector $\boldsymbol{p}$ perpendicular to the vertical plane containing the meteor; this plane is oriented at an angle $\psi$ to a horizontal reference direction $\mathrm{x}$.

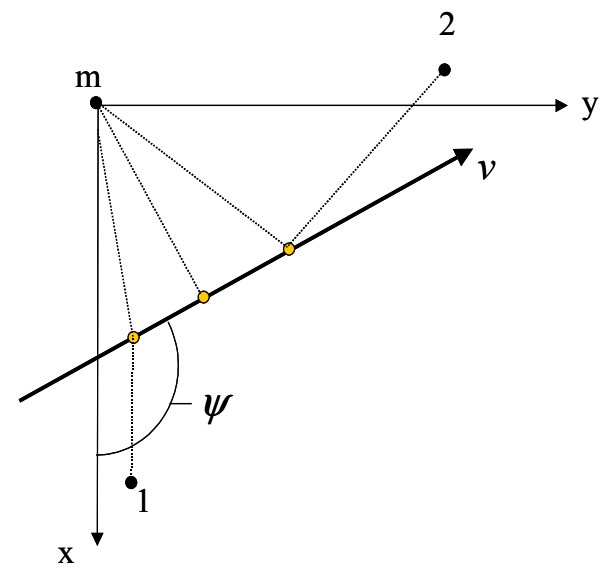

Fig. 6. A plan view of the meteor trail showing the specular reflection points from the transmitter to the 3 receiving sites $\mathrm{m}, 1$ and 2 (refer to Fig. 4).

are straightforward but the last one is more contentious and needs some care; further work on this aspect is desirable.

The basic idea behind the approach is illustrated in Fig. 5 . The direction from the main site to the meteor, unit vector $\boldsymbol{d}$, is known from the array measurements and this is perpendicular to the meteor train. The unit vector $\boldsymbol{p}$ is perpendicular to the vertical plane containing the meteor so that the direction of the meteor, $\boldsymbol{u}$, is simply given by the cross product of $\boldsymbol{d}$ and $\boldsymbol{p}$, i.e. $\boldsymbol{u}=\boldsymbol{d} \times \boldsymbol{p}$, which establishes the direction. What now remains is to determine the orientation of the vertical plane, i.e. the angle $\Psi$.

Figure 6 shows a plan view of the situation in Fig. 5 with the 3 -station arrangement relative to some arbitrary direction, $\mathrm{x}$ say. The time of observation of some characteristic point on the train, say the point of maximum increase in amplitude of the echo, leads to times $t_{1}, t_{2}$ and $t_{m}$ for the three stations.

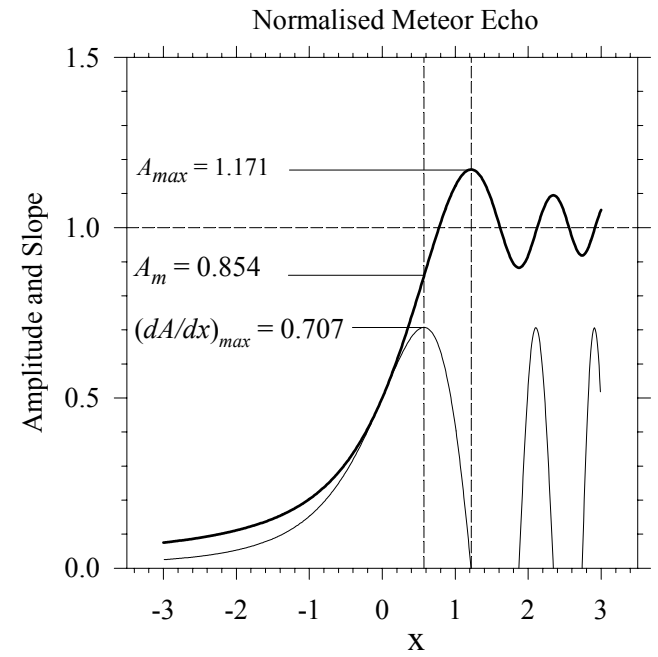

Fig. 7. Showing the classic rise in amplitude of a meteor echo on a back-scatter radar (heavy line) and the slope of the amplitude (light line). The abscissa is in terms of $x$, related to the physical distance $s$ along the trail by $s=F_{1} . x / \sqrt{ } 2$, where $F_{1}$ is the magnitude of the first Fresnel zone about the specular point.

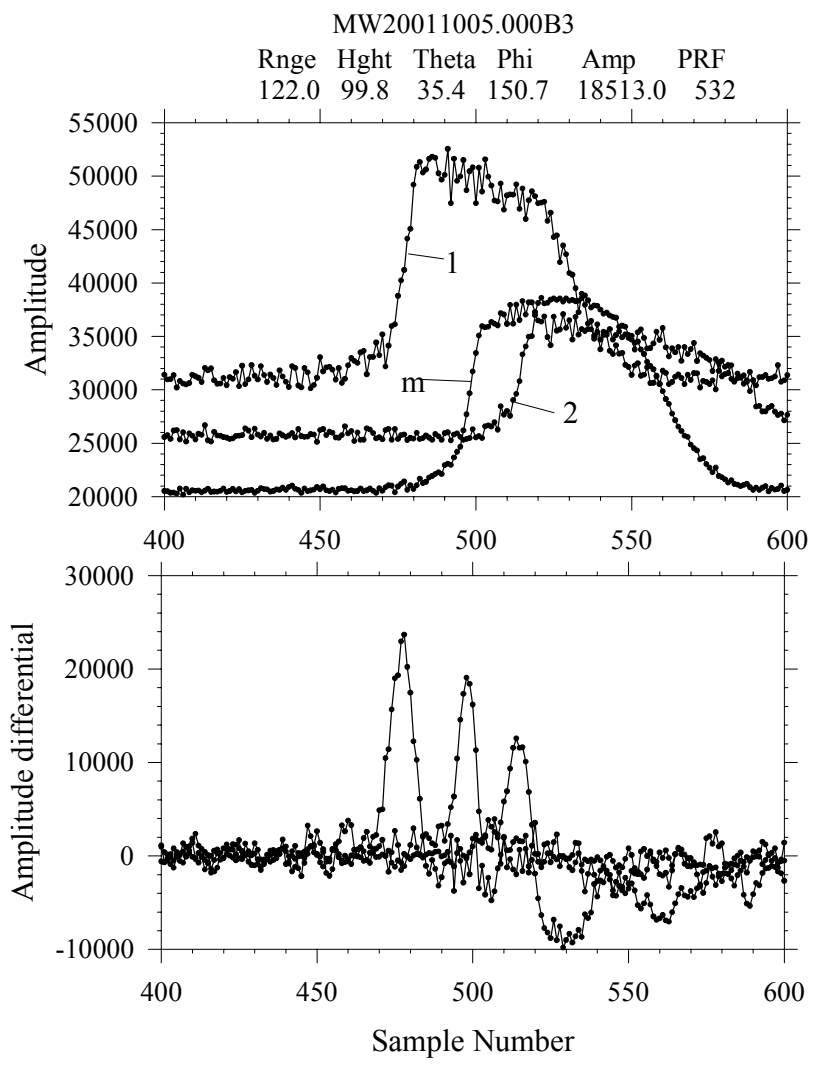

Fig. 8. A meteor echo observed on all three stations showing the amplitudes (offset for clarity) and derived slope. The time delays result in an estimate of $v=57.9 \mathrm{~km} . \mathrm{s}^{-1}$, while the rise-time gives a value of $61.9 \mathrm{~km} \cdot \mathrm{s}^{-1}$. 


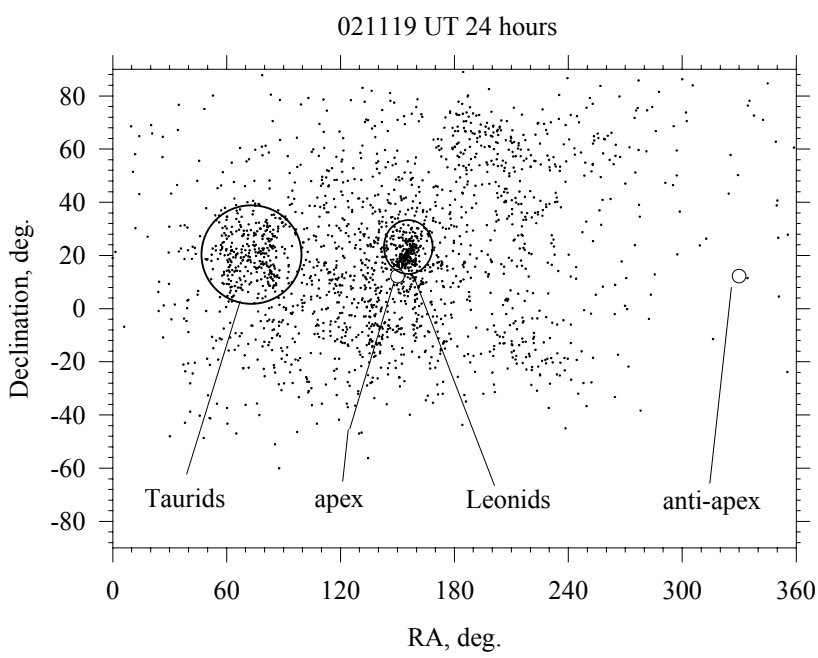

Fig. 9. The celestial coordinates of the radiants of the 2128 meteors observed by all 3 stations. The clustering around the expected value for the Leonids is apparent, as is the more diffuse radiant structure of the Taurids. The general sporadic background is a maximum in the direction of the Earth's way (the apex) and a minimum towards the anti-apex. Radiants below $-47^{\circ}$ in declination are not visible from this location.
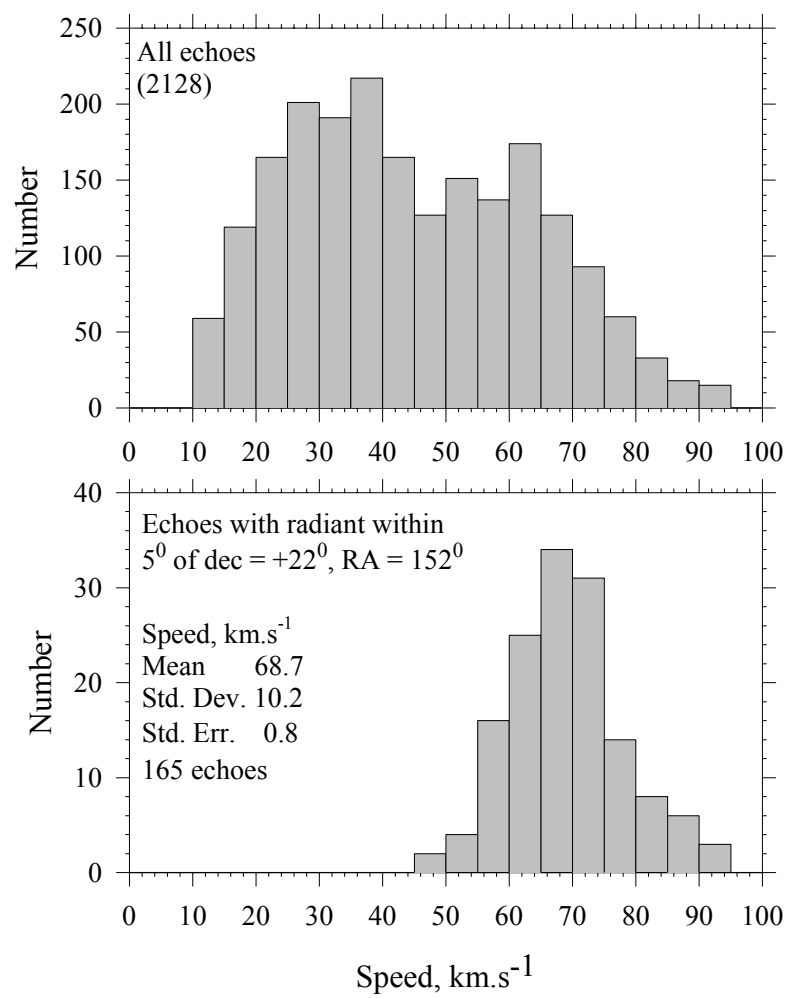

Fig. 10. The distribution of measured speeds for the 2128 echoes observed at the 3 stations and the speed distribution for radiants within $5^{\circ}$ of the expected Leonid radiant.
Labelling $T_{1}=t_{1}-t_{m}$ and $T_{2}=t_{2}-t_{m}$, the required angle, $\Psi$, is given by,

$\tan \psi=\frac{T_{2} x_{1}-T_{1} x_{2}}{T_{1} y_{2}-T_{2} y_{1}}$,

where the coordinates represent the positions of the two outer stations relative to the main station. The direction of the (horizontal) unit vector, $\boldsymbol{p}$, is now known so that the meteor direction, $\boldsymbol{u}$, is given by,

$\boldsymbol{u}=\boldsymbol{d} \times \boldsymbol{p}=u_{x} \boldsymbol{i}+u_{y} \boldsymbol{j}+u_{z} \boldsymbol{k}$,

and the speed, $v$, by,

$v=\frac{u_{x} \cdot\left(x_{1} y_{2}-x_{2} y_{1}\right)}{2 .\left(T_{1} y_{2}-T_{2} y_{1}\right)}=\frac{u_{y} \cdot\left(x_{1} y_{2}-x_{2} y_{1}\right)}{2 .\left(T_{1} x_{2}-T_{2} x_{1}\right)}$.

Depending on the orientation of the meteor, the appropriate version of Eq. (4) is used.

Alternative approaches for the determination of the speed from the data available from the 5 channels of the main receiver are possible. As the meteor echo develops, the rising amplitude gives estimates from (i) the phase of the echo prior to the point of orthogonality (the pre- $t_{o}$ approach), or (ii) the amplitude oscillations after this point as successive Fresnel zones are uncovered, or (iii) the rise-time itself. The last approach has proved to be most tractable and is illustrated in Fig. 7 in which the distance, $x$, is expressed in units of the first Fresnel zone relative to the specular point, i.e. distance in $\mathrm{km}, \mathrm{s}=F_{1} . x / \sqrt{ } 2$. The speed, $v$, can be estimated from the maximum slope and the maximum amplitude of the echo (Baggaley et al., 1997), i.e.

$v=\frac{d s}{d t}=\frac{1.657 \cdot p r f .(R \lambda)^{1 / 2}}{2 A_{\max }}\left(\frac{d A}{d n}\right)_{\max }$,

where $n$ is the sample number arising from the pulse repetition frequency $(p r f)$ at 1 sample/pulse, $(d A / d n)_{\max }$ is the maximum slope, $R$ is the range in $\mathrm{km}$ and $A_{\max }$ is the peak amplitude of the echo. The value of $A_{\max }$ sometimes can be hard to estimate if the echo is persistent and the train is distorted resulting in increased amplitude after the echo is established, especially if automatic computer routines are used. An alternative is to use the amplitude $A_{m}$ at the point of maximum slope so that

$v=\frac{1.212 \cdot p r f .(R \lambda)^{1 / 2}}{2 A_{m}}\left(\frac{d A}{d n}\right)_{\max }$.

\section{Experimental results}

An example of a meteor observed at all 3 stations is shown in Fig. 8; application of Eqs. (4) and (6) gives respective estimates of the speed, $v$, of 57.9 and $61.9 \mathrm{~km} . \mathrm{s}^{-1}$. In these estimates, the maximum slope position and value were estimated by fitting a parabola to the 3 adjacent points around 

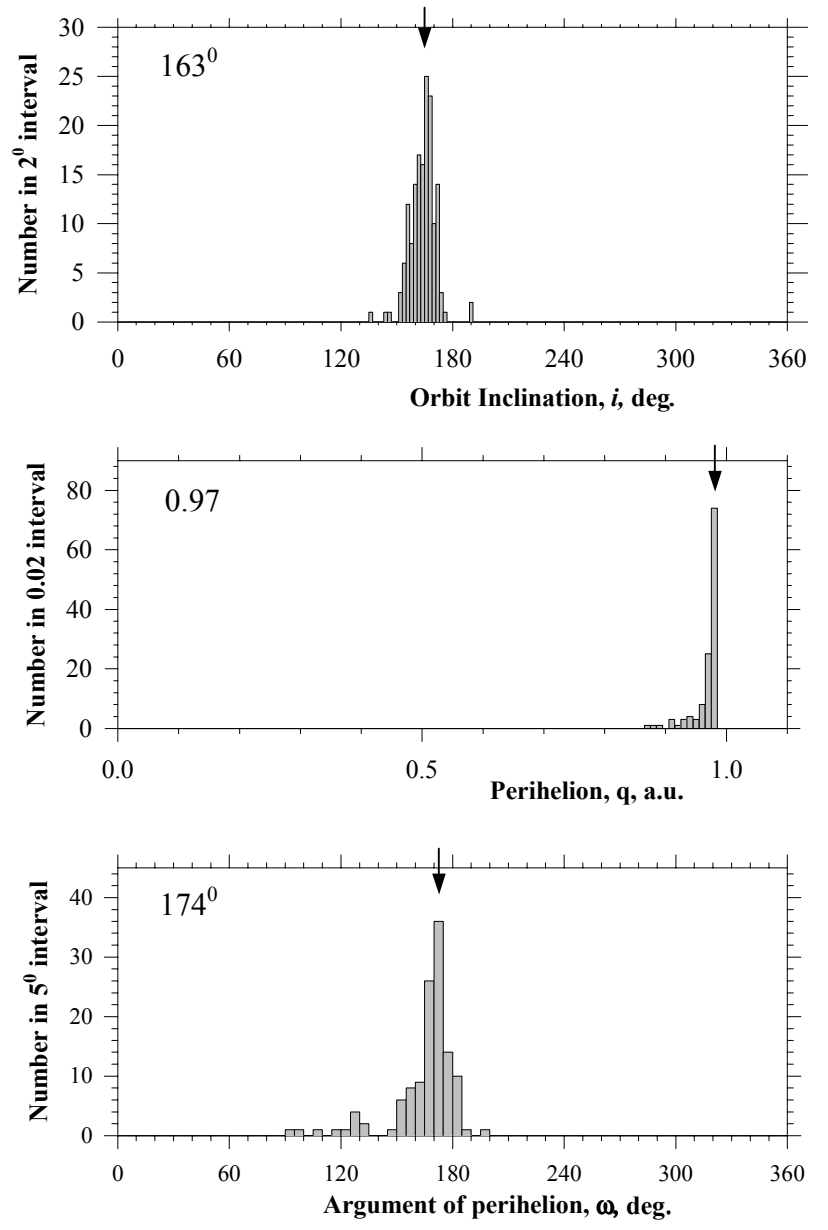

Fig. 11. Selected orbital elements for the 165 echoes with radiants within $5^{\circ}$ of the expected value. The nominal values are as indicated.

the peak. Another approach is to determine the position of the centroid of the points around the peak, which gives comparable answers and has been used extensively in the automated software.

Estimated orbital parameters are generated automatically and here we examine data taken on 19 November 2002 during the Leonid shower. The time interval used was 00:00 to 24:00 UT and the observed peak in activity was about $1 \mathrm{~h}$ in extent centered at about 10:40 UT. The total number of echoes observed during this 24 -h period was 8435 as shown in Fig. 3 above. Of these, 2128 echoes were captured also on "both" of the outlying stations allowing an estimate of the speed and orbital parameters.

A plot of the radiant position for each of the above 2128 echoes is shown in Fig. 9. The clustering of echoes around the nominal Leonid radiant (declination $22^{\circ}$, right ascension $152^{\circ}$ ) is apparent. A second more diffuse clustering is consistent with the expected Taurid radiant, which is active for most of November. Selecting meteors that are within $\pm 5^{\circ}$ of

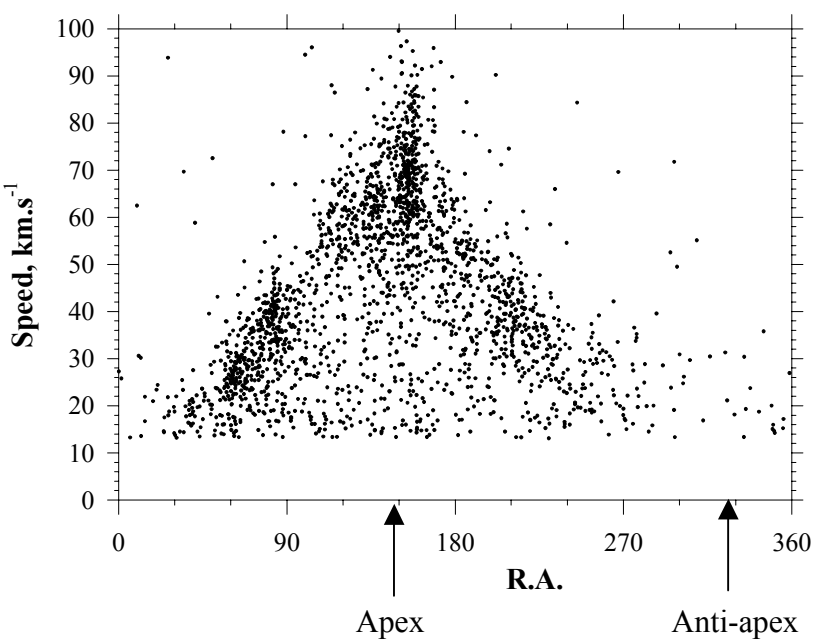

Fig. 12. The distribution in speed versus R.A. for the 2128 echoes seen on all 3 stations. The Leonid and Taurid radiant again are apparent, as is the expected generally increased speed in the direction of the apex.

the nominal Leonid radiant results in 165 such echoes. The distribution in speed for these 165 echoes is shown in Fig. 10, giving an estimate of $68.7 \pm 0.8 \mathrm{~km} . \mathrm{s}^{-1}$, consistent with the accepted value; the distribution of speeds for all echoes on that day are also shown for comparison.

Distributions of orbital inclination $(i)$, perihelion distance $(q)$ and argument of perihelion $(\omega)$ are shown in Fig. 11 for the 165 "Leonid" meteors along with the nominal values (see McKinley, 1961). The agreement is apparent.

The distribution of speeds for the 2128 3-station meteors as a function of the Right Ascension (R.A.) is shown in Fig. 12, where the clustering associated with the Leonids and Taurids is again apparent.

\section{Discussion}

The system described here provides basic positional data for meteors observed at the main station to an accuracy of $\sim 3 \mathrm{~km}$ in range, set by the sampling rate of $50 \mathrm{ksps}$, and $1^{\circ}$ in angular resolution in elevation and azimuth from the 5-antenna spacings. The addition of the outlying stations allows access to the velocity of the meteoroid in magnitude and direction. The system is capable of handling simultaneous echoes provided that they occur in different range bins; the occurrence of simultaneous echoes at the same range is extremely rare.

In determining the orbital elements of individual meteoroids from the 3 -station measurements, it is clear that a good estimate of some of the elements is provided. Others though, notably the eccentricity $(e)$ and the semi-major axis $(a)$, are very dependent on the estimate of the speed of the meteor. The fairly wide spread (standard deviation $\sim 10 \mathrm{~km} . \mathrm{s}^{-1}$ ) in the speed distribution of the meteors within 
$5^{\circ}$ of the Leonid radiant may be partly due to the fact that a few non-Leonids will be included, but mostly due to the inherent uncertainty in the estimate. This is further illustrated by the relatively large number of apparently hyperbolic meteors $\left(v>72 \mathrm{~km} . \mathrm{s}^{-1}\right)$, shower and sporadic alike, in Fig. 12, which is not interpreted as a true representation.

Nevertheless, the results are encouraging and techniques for improving the estimates of the various quantities, especially the speed, are being pursued. These include improved signal processing and estimation of the time difference between the echoes at the 3 stations. The 3-station system operates on a continuous 24-h basis and the raw data are stored on DVDs, allowing the introduction of these improved techniques as they are developed.

Acknowledgements. The authors would like to acknowledge the invaluable assistance of Z. Krzeminski and R. Weryk.

Edited by: D. Janches

\section{References}

Baggaley, W. J., Bennett, R. G. T., and Taylor, A. D.: Radar meteor atmospheric speeds determined from echo profile measurements, Planet. Space Sci., 45, (5), 577-583, 1997.

Campbell-Brown, M. and Jones, J.: Determining the initial radius of meteor trains: fragmentation, Monthly Notices of the Royal Astronomical Society, 343, 3, 775-780, 2003.

McKinley, D. W. R.: Meteor Science and Engineering, McGrawHill, 151, 1961.

Hawkins, G. S.: Meteors, Comets and Meteorites, McGraw-Hill, 44, 1964.

Jones, J., Webster, A. R., and Hocking, W. K.: An improved interferometer design for use with meteor radars, Radio Sci., 33, (1), 55-65, 1998. 